\title{
AUDITORÍA INTERNA COMO GESTOR DE OPTIMIZACIÓN EN LOS SERVICIOS PÚBLICOS: SEGURO SOCIAL DE SALUD DEL PERÚ
}

\author{
INTERNAL AUDIT AS MANAGER OF OPTIMIZATION IN PUBLIC SERVICES: \\ SEGURO SOCIAL DE SALUD DEL PERÚ
}

LiLIANA Ruth HuAmán Rondón Universidad del Callao Lima, Perú

ORCID: https://orcid.org/0000-0002-0280-4227 Correo electrónico: lilianarhr27@gmail.com

\section{RESUMEN}

Objetivo: Determinar si la auditoría interna constituye un agente importante que permita optimizar los procesos de calidad de los servicios asistenciales que presta Essalud en el Perú. Método: Es una investigación de tipo descriptivo, transversal, prospectivo, analítico, correlacional y de diseño no experimental. Se aplicó una encuesta sobre los controles de los servicios de salud a 384 usuarios del Hospital Nacional Edgardo Rebagliati Martins (Lima), del Hospital Nacional Guillermo Almenara Irigoyen (Lima) y del Hospital IV Alberto Sabogal Sologuren (Callao). Resultados: Se encontró que los exámenes especiales de Auditoría interna tuvieron un $57,5 \%$ de efectividad en el período de estudio 2013 2016. Conclusiones: Se confirmó la necesidad de contar con los informes de auditoría sobre la gestión de calidad del servicio que brindan las entidades de salud.

Palabras clave: Auditoría; optimización; gestión; calidad; servicio; salud.

\begin{abstract}
Objective: Determine if the internal audit is an important agent that allows to optimize the quality processes of the health services provided by Essalud in Peru. Method: It is a descriptive, transversal, prospective, analytical, correlational and non-experimental design research. A survey on the controls of health services was applied to 384 users of the Edgardo Rebagliati Martins-Lima National Hospital, Guillermo Almenara Irigoyen-Lima National Hospital, and IV Alberto Sabogal Sologuren-Callao Hospital. Results: It was found that the special Internal Audit exams had $57.5 \%$ effectiveness of the 2013-2016 study period. Conclusions: We confirm the need to have the audit reports on quality management of the service provided by health entities.
\end{abstract}

Keywords: Audit; optimization; management; quality; service; health. 


\section{INTRODUCCIÓN}

La política de salud en el Perú incluye el financiamiento, la asistencia sanitaria, la salud pública, la salud preventiva, las enfermedades crónicas, la discapacidad, la atención a largo plazo y la salud mental, según se declara en el D.S.027-2007-PCM (Presidencia del Consejo de Ministros, 2007, p. 8) que define las políticas nacionales y establece su estricto cumplimiento; luego, el referido decreto fue modificado con el D.S.025-2010-PCM y se incluye la entrega de servicios de calidad a los usuarios de los principales hospitales como el Hospital Nacional Edgardo Rebagliati Martins, el Hospital Nacional Guillermo Almenara Irigoyen y el Hospital IV Alberto Sabogal con un total de 4944672 usuarios en ellos a junio del 2014, según Gerencia Técnica - Gerencia Central de Aseguramiento EsSalud -2014" (Gerencia Técnica Gerencia Central de Aseguramiento, 2014); sin embargo, para su mejor desempeño se efectúan acciones de auditoría interna cuyo propósito funcional es orientar la gestión administrativa de las entidades del Seguro de Salud del Perú (EsSalud). La Contraloría General de la República, como ente rector de las acciones de control gubernamental, imparte normas de cumplimiento administrativo y operativo con la posibilidad de que se pueda optimizar la actividad de salubridad pública. Los usuarios de los establecimientos de salud requieren mayores atenciones del servicio de salud; es deseable el incremento de los servicios en general tanto para elevar los niveles de atención de calidad, así como para ampliar la cobertura. Se ha medido la percepción de los usuarios sobre los servicios de salud para que los directivos puedan tomar acciones correctivas en cuanto a rediseñar los procesos tendientes a optimizar la gestión de la calidad de los servicios de salud.

Hace muchos años EsSalud afronta serios problemas en la prestación de servicios a los asegurados, producto de una deficiente gestión que a la fecha no ha superado sus carencias, como: inadecuados controles de auditoría interna, escasa presencia del órgano de control, la deficiente e inoportuna atención de los servicios de salud reflejado en las largas colas y lentas en su proceder. Los asegurados se quejan por el sistema de atención y el otorgamiento de citas, la falta de interés y amabilidad del personal, y una buena proporción de asegurados se encuentran insatisfechos con la provisión de medicamentos. Mientras, la población crece a un ritmo de 1,3\% anual (Instituto Nacional de Estadística e Informática [INEI], 2014), la oferta de servicios no ha crecido al mismo ritmo, con una población de 31151643 habitantes en el Perú (INEI, 2016) y 10754665 de asegurados. La cobertura de atención de la prestación de servicios se encuentra en el orden del 34,5\% de los asegurados (INEI, 2016), pues es de verse que el déficit de cobertura y atención se encuentra muy elevado.
Es en ese sentido que EsSalud, como entidad prestadora de servicios de salud pública, se encuentra ligada a los controles gubernamentales que evalúan las decisiones ejecutivas y administrativas conforme a las normas que le permiten desarrollar en la dinámica de sus funciones; en consecuencia, las cuestiones administrativas son una limitante para la atención oportuna o apropiada de los asegurados y es la auditoría interna una herramienta de control importante para la optimización del proceso de sus atenciones, motivo que sustenta la investigación.

Partiendo del Sistema Nacional de Control (SNC), como fundamento del encargo constitucional que el Estado le da a través del Artículo $82^{\circ}$ que a la letra dice:

La Contraloría General de la República es una entidad descentralizada de Derecho Público, goza de autonomía conforme a su ley orgánica. Es el órgano superior del Sistema Nacional de Control que supervisa la legalidad de la ejecución del Presupuesto del Estado, de las operaciones de la deuda pública y de los actos de las instituciones sujetas a control. (Congreso Constituyente Democrático [CCD], 1993)

La propia Contraloría General y las Sociedades de Auditoría pueden efectuar auditorías externas y el Órgano de Control Institucional (OCI) de la propia entidad debe efectuar auditorías financieras y de gestión, así como exámenes especiales y otras acciones permitidas por las normas y regulaciones que la Ley de Control establece en aplicación de los criterios de gestión, que permitirán la emisión de informes para el mejoramiento de la gestión.

El principal objetivo del ente rector ha sido supervisar la legalidad de la ejecución presupuestal de las entidades del Estado, informando mayormente sobre la irregular o ilegal administración de los fondos públicos, sin observar si los recursos públicos se ejecutan en el marco de una gestión en beneficio de la población y del objetivo predeterminado, es decir, no informando mayormente sobre el comportamiento y desempeño de la gestión de las instituciones sujetas a control.

El sistema dispone de normas específicas para su aplicación por el personal de cada órgano que lo conforma, entre estas tenemos "normas, procedimientos y principios que regulan el control gubernamental, efectúa la verificación y evaluación, objetiva y sistemática, de los actos y resultados producidos por la entidad en la gestión y ejecución de los recursos, bienes y operaciones institucionales" (Ley 27785, 2002, art. 10), además comprende auditorías internas y exámenes especiales que deben realizarse "en función a la: Misión de la entidad, los Criterios de Rotación de Énfasis, Materialidad, Áreas Críticas y/o de incidencia, requerimientos provenientes de Directivas específicas y/o normas 
de carácter general, y demás disposiciones contenidas en la normativa de control vigente". (UNMSM, 2016). "Así como en el desempeño de la entidad vinculado a la previsión de servicios públicos y al logro de resultados en beneficio del ciudadano" (p.1) Resolución de Contraloría No 418-2013CG. (Contraloría General de la República, 2013)

El Instituto de Auditores Internos (2004) ha elaborado las siguientes definiciones de auditoría interna, aceptada mundialmente:

Auditoría interna es una actividad independiente $y$ objetiva de aseguramiento y consulta concebida para agregar valor y mejorar las operaciones de una organización. Ayuda a una organización a cumplir sus objetivos aportando un enfoque sistemático y disciplinado para evaluar y mejorar la eficacia de los procesos de gestión de riesgos, control y gobierno. CP 1000.C1-1 (IIA I. d.-I.-2., 2004)

La independencia está establecida por la estructura de la organización y las líneas de reporte. La objetividad se logra mediante una apropiada actitud mental. La actividad de auditoría interna evalúa la exposición al riesgo del gobierno, las operaciones y los sistemas informáticos de la organización, con respecto a lo siguiente: Eficacia y eficiencia de las operaciones, confiabilidad e integridad de la información financiera y operativa, protección de activos y cumplimiento de leyes, regulaciones y contratos. (IIA T. I., 2004)

Sobre esta actividad, los autores Eugenio y Lozano (2014) afirman que la Auditoría interna de gestión:

Es el examen y evaluación que se realiza a los procesos, programas y planes de una entidad, a fin de observar su nivel de cumplimiento y determinar el grado de eficacia, eficiencia y economicidad en el manejo de recursos y si los resultados obtenidos permiten alcanzar las metas y objetivos planteados. (p. 21)

Hamui, Fuentes, Aguirre y Ramírez (2013), en el estudio sobre la satisfacción de la calidad de las atenciones en México, describen que:

El Sistema Nacional de Salud es el bienestar de la población y su estado saludable. Una sociedad en la que sus miembros viven enfermos, física o mentalmente, tiene menos bienes relacionales, baja de productividad, aumento de gastos y mengua en el rendimiento económico, pero sobre todo presenta la disminución del bienestar subjetivo, la satisfacción y la felicidad. De ahí que, el estudio de la relación entre la atención médica, que busca restaurar, mantener y/o fomentar los estados saludables y la evaluación que las personas realizan sobre su propia circunstancia en el encuentro con las instituciones de salud, sea de interés para comprender algunas de las necesidades de ciertos grupos de población. (p. 21)

Prosiguen Hamui et al.(2013) afirmando que:

Uno de los retos conceptuales de este estudio es la definición de la noción de satisfacción. A diferencia de los indicadores objetivos, como el PBI y el ingreso, los indicadores subjetivos como la satisfacción y la felicidad están ligados al estado de bienestar de las personas, no obstante, por la variedad de significados que el bienestar previsiblemente implica, no puede ser imputado ni predeterminado desde fuera. En este sentido, como explica Millán, los indicadores subjetivos correctamente diseñados, son muy cercanos al objeto que pretenden medir. Al dar como válida la propia evaluación, se evita dotar al bienestar de una visión trascendental que suprima la experiencia concreta del individuo, de ahí que lo importante sea determinar las condiciones en las que se realiza la evaluación individual del bienestar y no desecharla por subjetiva. La propia evaluación de la vida, o de algún ámbito de la experiencia, (la familia, la atención a la salud, etc...). Habría que aclarar que la objetividad de un indicador se refiere al incremento de posibilidad de que una evaluación hecha por un tercero obtenga el mismo resultado del primero. Los datos sobre percepciones tienen, al menos, un carácter binario: si o no. La posibilidad de que la primera evaluación no coincida con la segunda es más amplia, por lo que la información resulta más dispersa $y$ variable. (p. 23)

El SERVQUAL (deriva de los términos service quality) es el instrumento más usado por las organizaciones para medir la calidad de los servicios y la satisfacción del cliente. Fue creado en los años 80 en Estados Unidos por Parasuraman, Zeithaml y Berry. Consiste en evaluar expectativas y percepciones de los clientes o usuarios de una entidad, considerando cinco dimensiones (elementos tangibles, fiabilidad, capacidad de respuesta, seguridad, empatía) y veintidós parámetros sobre calidad del servicio, entre los cuales mencionamos: equipos y tecnología médica de punta, tiempo de atención antes durante y después del servicio, rapidez en el servicio, la seguridad en la atención del servicio y los horarios flexibles de atención (Piedra-Valoy y Lipa-Chancolla,2018).

\section{MATERIALES Y MÉTODOS}

Es una investigación de tipo descriptivo, transversal, prospectivo, analítico, correlacional y de diseño no experimental. Se aplicó una encuesta sobre los controles 
de los servicios de salud a 184 usuarios del Hospital Nacional Edgardo Rebagliati Martins(Lima), del Hospital Nacional Guillermo Almenara Irigoyen (Lima) y del Hospital IV Alberto Sabogal Sologuren (Callao). A partir de la exploración de los parámetros de calidad de la atención de los servicios de salud que prestan las entidades consideradas en el estudio y asimismo determinar su nivel de satisfacción (Zas, 2002).

\section{RESULTADOS}

Se ha investigado a los usuarios representativos de los 3 principales nosocomios de EsSalud, los detalles se reflejan en la tabla 1.

La unidad de análisis estuvo comprendida por los usuarios asegurados del sistema EsSalud. Se debe distinguir según la muestra, que existen 31151643 habitantes en el país, de los cuales se da cobertura a 10754665 de asegurados; de esa proporción se atiende únicamente al $34,5 \%$, por tanto, se evidencia un déficit de atenciones del $73,5 \%$ que es una porción mayoritaria (ver Figura 1).

Si observamos la atención efectiva, por cada 1000 asegurados se dispone de 0,78 camas, un índice poco halagador para el sistema (ver Figura 2)

A continuación, en la Tabla 2 se presenta el tratamiento descriptivo y análisis de los niveles de satisfacción que perciben los 384 asegurados de EsSalud.

Como se muestra en la tabla 2 y la figura 3 , respecto a la variable calidad del servicio, los usuarios reflejaron su percepción sobre la calidad del modo siguiente: el 45,83\% se encuentra satisfecho; el $31,51 \%$ se siente insatisfecho;

Tabla 1

Población de asegurados en los 3 hospitales de Lima y Callao, 2014.

\begin{tabular}{|c|c|c|c|}
\hline HOSPITAL & Población Usuarios & $\%$ & Muestra \\
\hline 1. Hospital Nacional Edgardo Rebagliati Martins - Lima & 1790015 & 36,20 & 139 \\
\hline 2. Hospital Nacional Guillermo Almenara Irigoyen - Lima & 1511416 & 30,57 & 117 \\
\hline 3. Hospital IV Alberto Sabogal Sologuren- Callao & 1643241 & 33,23 & 128 \\
\hline TOTAL & 4944672 & 100,00 & 384 \\
\hline
\end{tabular}

Fuente: Gerencia Técnica. Gerencia Central de Aseguramiento EsSalud -2014 (CONAREME, 2016). Elaboración propia

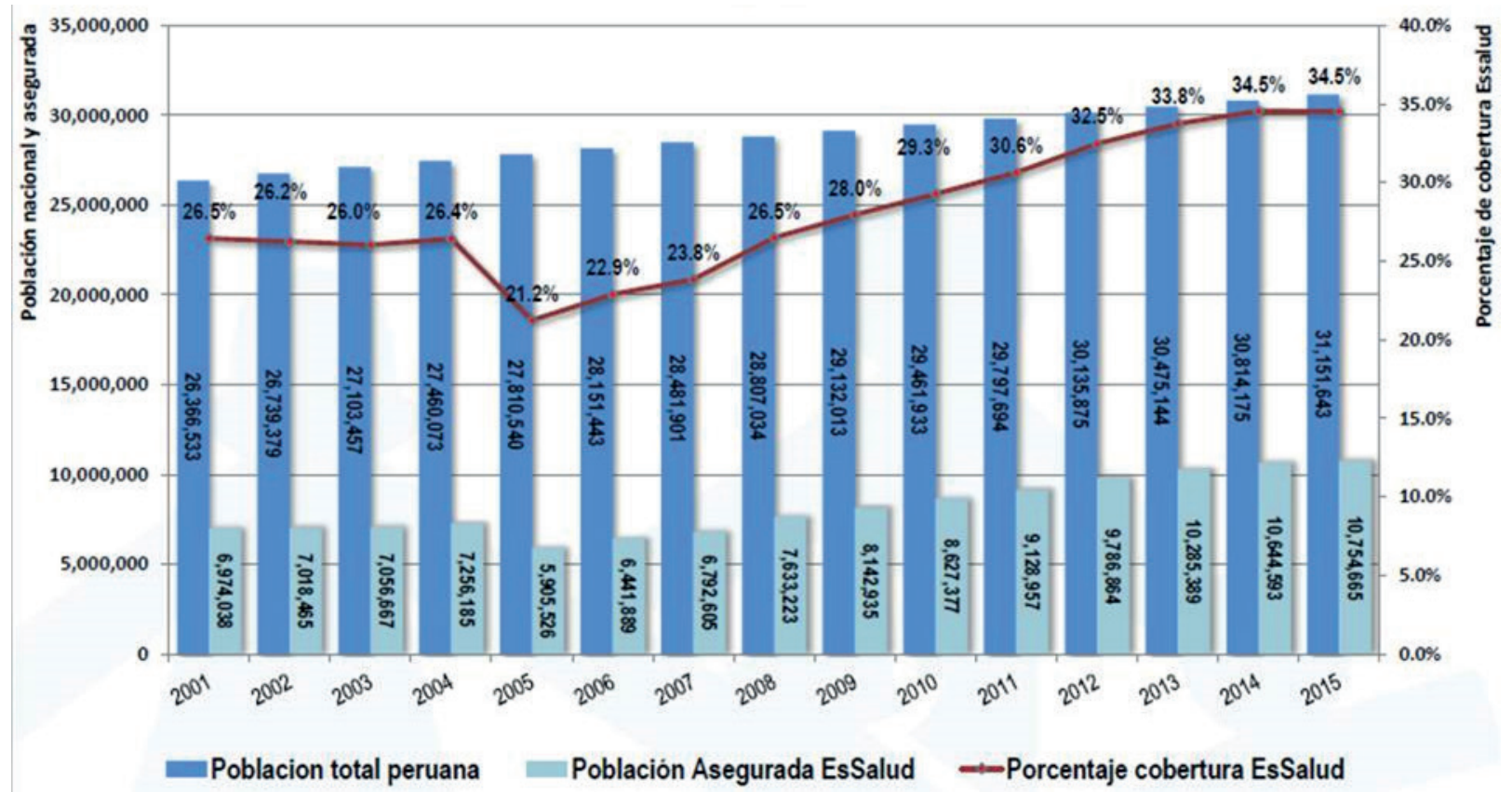

Figura 1. Porcentaje de cobertura de asegurados de ESSALUD respecto a la población total peruana (2001-2015). Fuente: INEI 
Tabla 2

Niveles de satisfacción sobre la calidad del servicio en EsSalud

\begin{tabular}{lcc}
\hline Niveles & Frecuencia & Porcentaje \\
\hline No aplica & 4 & 1,0 \\
Muy insatisfecho & 35 & 9,1 \\
Insatisfecho & 121 & 31,5 \\
Satisfecho & 176 & 45,8 \\
Muy satisfecho & 48 & 12,5 \\
Total & 384 & 100,0 \\
\hline
\end{tabular}

Fuente: elaboración propia

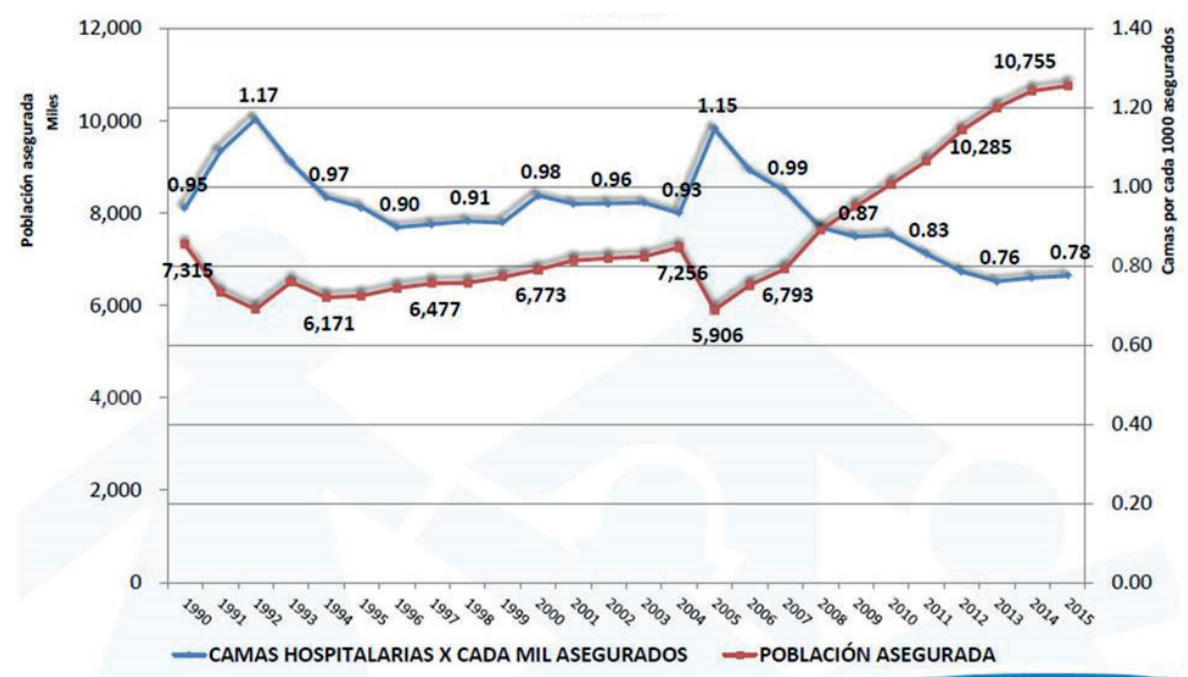

Figura 2. Población asegurada en miles de camas hospitalarias por cada mil asegurados (1990-2015). Fuente: Boletín Nacional Estadístico.

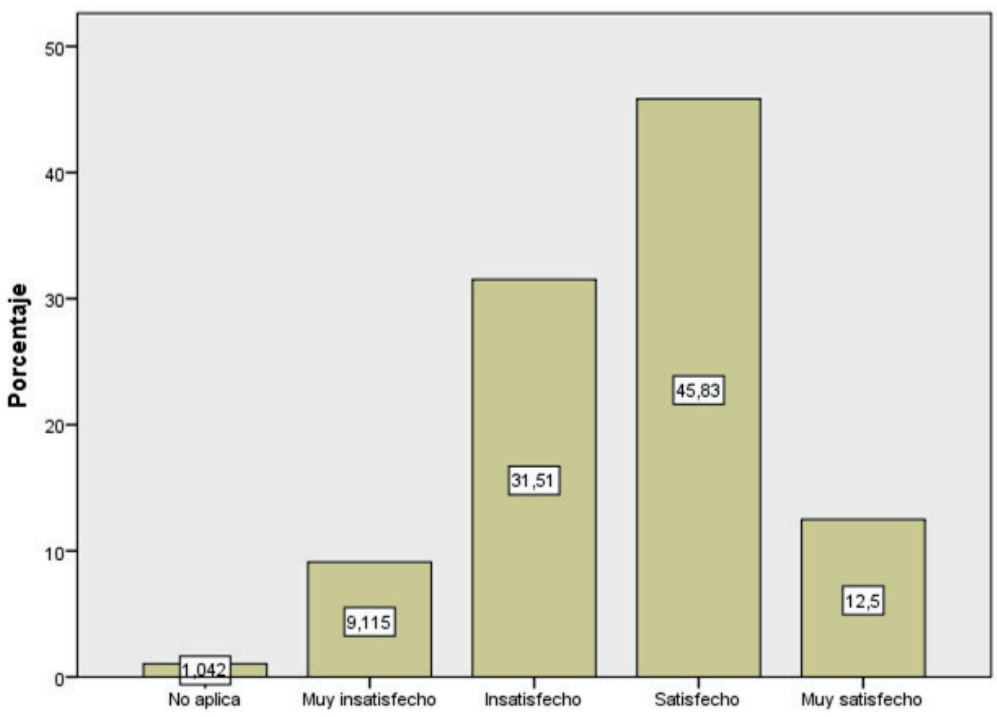

Figura 3. Niveles de satisfacción sobre la calidad del servicio en EsSalud (\%) Fuente: Elaboración propia. 
el 12,5\%, muy satisfecho; el 9,1\%, muy insatisfecho; y el $1 \%$ no aplica; lo que hace inferir que hay ligeramente un porcentaje mayor de estar satisfecho aun cuando se presenta malestar en los usuarios por la atención en general.

Se muestra en la tabla 3 y Figura 4, que la variable auditoría interna supone realizar actividades de control que aun cuando muchos usuarios comprenden que es una labor necesaria responden por dicho trabajo. Así el 42,45\% considera que es deficiente; el $31,51 \%$ opina que es regular; y el $26,04 \%$ cree que el trabajo de la auditoría interna es excelente; por tanto, se colige que se debe continuar impulsando labores de control para promover una buena gestión en los hospitales donde se ha realizado el estudio y lograr su optimización.

Resultados de la correlación de Spearman

Tipo de estudio:

Transversal

Nivel de investigación: Relacional

Objetivo estadístico: Correlacional
Variable de estudio: Ordinal - Ordinal

Tabla 3

Niveles de satisfacción sobre el trabajo de Auditoría Interna en los hospitales de EsSalud.

\begin{tabular}{lcc}
\hline Niveles & Frecuencia & Porcentaje \\
\hline Deficiente & 163 & 42,4 \\
Regular & 121 & 31,5 \\
Excelente & 100 & 26,0 \\
Total & 384 & 100,0 \\
\hline
\end{tabular}

Fuente: Elaboración propia

Se desea correlacionar si la auditoría interna es efectiva para alcanzar el mejoramiento de la calidad en los servicios que brinda EsSalud (Ver tabla 4).

Las hipótesis planteadas son las siguientes:

$\mathrm{H}_{0}$ : Los niveles de calidad de los servicios asistenciales de EsSalud no son dependientes de la aplicación oportuna de la Auditoría Interna.

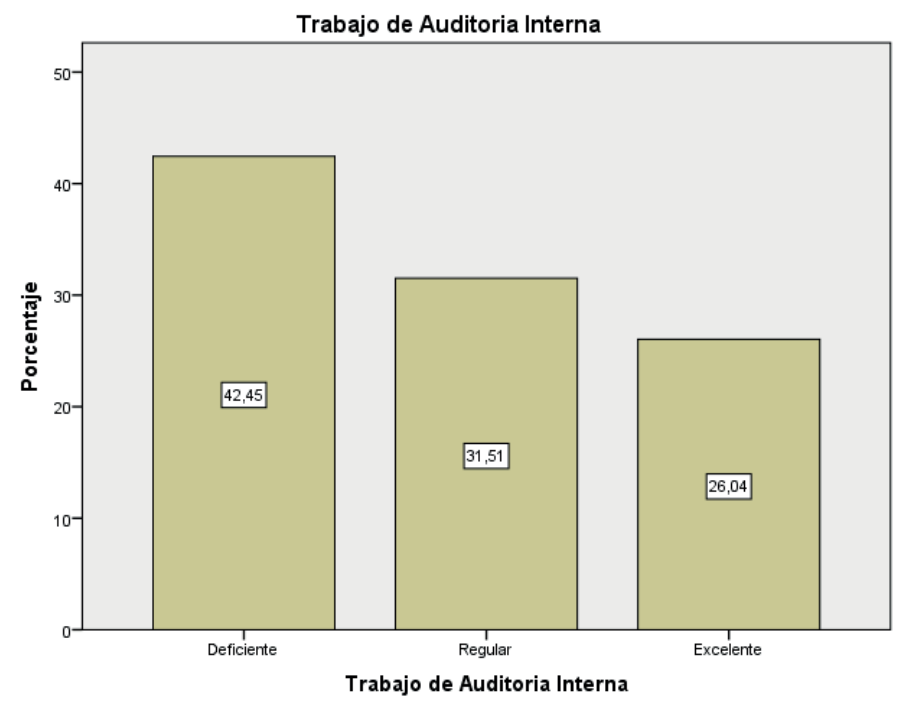

Figura 4. Niveles de satisfacción sobre el trabajo de Auditoría Interna en los hospitales de EsSalud (\%) Fuente: Elaboración propia.

Tabla 4

Tabla de contingencia entre la Auditoría Interna y la Calidad de los servicios de EsSalud.

\begin{tabular}{|c|c|c|c|c|c|c|}
\hline \multirow{2}{*}{ Auditoría Interna } & \multicolumn{6}{|c|}{ Calidad de los servicios de EsSalud } \\
\hline & Muy en desacuerdo & En desacuerdo & Indeciso & De acuerdo & Muy de acuerdo & Total \\
\hline $\mathrm{n}$ & 4 & 35 & 121 & 176 & 48 & 384 \\
\hline Media & 3 & 3 & 2 & 2 & 3 & 2 \\
\hline Desviación Estándar & 0,00 & 0,65 & 1,00 & 0,87 & 0,00 & 0,91 \\
\hline Error típico & 0,00 & 0,11 & 0,09 & 0,07 & 0,00 & 0,05 \\
\hline
\end{tabular}

Fuente: Elaboración propia 
$\mathrm{H}_{1}$ : Los niveles de calidad de los servicios asistenciales de EsSalud son dependientes de la aplicación oportuna de la Auditoría Interna.

Establecer un nivel de significancia: Nivel de significancia a $($ alfa $)=5 \%$ o 0,05

Seleccionar estadístico de prueba: Correlación de Spearman (Rho) (Ver Tabla 5)

Interpretación: Valor de "Rho" calculado 0,148

Valor de $\mathrm{p}=0,004$

(El error de la muestra está por debajo de 0,05 por tanto no es significativa)

Decisión: Rechazamos $\mathrm{H}_{0} \mathrm{y}$ aceptamos $\mathrm{H}_{1}$.

$\mathrm{H}_{1}$ : Los niveles de calidad de los servicios asistenciales de EsSalud son dependientes de la aplicación oportuna de la Auditoría Interna.

Es una correlación positiva baja, por lo que hay una escasa relación y habría otros factores que estarían influyendo en la calidad del servicio de salud.

\section{DISCUSIÓN}

La sociedad peruana requiere que la prestación de servicios sea de calidad. En efecto, medir la calidad en la prestación de servicios de salud hace posible tener expectativas por las revelaciones que generan en los usuarios. Vargas, Valecillos y Hernández (2013) refieren que se debe poner especial cuidado a la calidad de atención, tomando en cuenta tanto las expectativas como la satisfacción de los usuarios. La deficiencias en la prestación de servicios de salud generan maletar en los usuarios, lo cual se constata con el $62 \%$ de rechazo por EsSalud. La investigación concluye claramente que, en las actuales circunstancias, la aplicación de la auditoría interna contribuye a optimizar la gestión de los servicios que brinda EsSalud, siendo una actividad imprescindible que sirve para equilibrar la ejecución de tareas en salud, la custodia y buena administración de los recursos aportados por los empleadores y los trabajadores. La incidencia de la Auditoría Interna en EsSalud, según la percepción de los usuarios encuestados, tiene un acumulado de efectividad del 57,5\% entre excelente y regular, lo que confirma la necesidad de contar con los informes de auditoría sobre la gestión de calidad del servicio que prestan las entidades de salud; es más, se estaría dando cumplimiento al Artículo $15^{\circ}$ inc. c: Impulsar la modernización y el mejoramiento de la gestión pública, a través de la optimización de los sistemas de gestión y ejerciendo el control gubernamental con especial énfasis en las áreas críticas sensibles de la Ley № 27785, mediante sus recomendaciones, cumpliendo así el rol de gestor de optimización para la calidad de los servicios.

\section{REFERENCIAS}

Constitución Política del Perú 1993. Lima, Perú. Obtenido de http://www.congreso.gob.pe/Docs/files/documentos/constitucionparte1993-12-09-2017.pdf

EsSalud (2016). EsSalud. Obtenido de:http://www.essalud.gob.pe/doctor-alvaro-vidal-asumio-como-presidente-ejecutivo-de-essalud/

Eugenio, K. y Lozano, L. (2014). Diseño del Departamento de Auditoría Interna de la Benemérita Sociedad Protectora de la Infancia Hospital León Becerra de la Ciudad de Guayaquil. Guayaquil, Ecuador: Universidad Politénica Salesiana sede Guayaquil. Obtenido de: www. dspace.ups.edu.ec acosta e. \& heredero L.

Gerencia Técnica Gerencia Central de Aseguramiento, E. (2014). Distribución de población asegurada activa

Tabla 5

Coeficiente de correlación Rho de Spearman entre la Auditoría Interna y la Calidad de los servicios de EsSalud

\begin{tabular}{|c|c|c|c|c|}
\hline \multicolumn{5}{|l|}{ Correlaciones } \\
\hline & & & Auditoría Interna & Calidad del Servicio \\
\hline \multirow{6}{*}{ Rho de Spearman } & \multirow{3}{*}{ Auditoría Interna } & Coeficiente de correlación & 1,000 & $148^{* *}$ \\
\hline & & Sig. (bilateral) & . &, 004 \\
\hline & & $\mathrm{N}$ & 384 & 384 \\
\hline & \multirow{3}{*}{ Calidad del Servicio } & Coeficiente de correlación & $148^{* *}$ & 1,000 \\
\hline & & Sig. (bilateral) &, 004 & . \\
\hline & & $\mathrm{N}$ & 384 & 384 \\
\hline
\end{tabular}

**. La correlación es significativa al nivel 0,01 (bilateral)

Fuente: Elaboración propia 
por redes asistenciales - junio 2014 . Lima, Perú. Obtenido de: www.essalud.gob.pe

Gonzáles, J. y Pazmiño, M. (2015). Cálculo e interpretación del Alfa de Cronbach para el caso de validación de la consistencia interna de un cuestionario, con dos posibles escalas de Likert. Revista Publicando, 1-15. Obtenido de https://www.ssoar.info/ssoar/bitstream/handle/document/42382/ssoar-revpublicando-2015-1-gonzalez_alonso_jorge_et_al-Calculo_e_ interpretacion_del.pdf? sequence $=1$

Hamui, L., Fuentes, R., Aguirre, R., y Ramírez, O. (2013). La satisfacción/insatisfacción de los usuarios del sistema de salud con la atención desde sus expectativas y experiencias en México. (V. S. Especialidad, Ed.) México. Distrito Federal, México: Universidad Nacional Autónoma de México. Obtenido de http://www.facmed. unam.mx/bmnd/SatisfaccionAtencionMedica.pdf

Hernández, D. (2007). Perú: la auditoría de gestión en el área de recursos humanos de EsSalud. Lima: www.monografías.com. Obtenido de http://www.upt.edu.pe/ ouci/archivo/2010\%20-\%20LA\%20AUDITORIA\%20 ACADEMICA\%20PARA\%20LA\%20APROPIADA\%20GESTION\%20EN\%20UNA\%20INSTITUCION\%20EDUCATIVA\%20DE\%20NIVEL\%20SUPERIOR.pdf

Hernández, R., Fernández, C., y Baptista, P. (2014). Metodología de la investigación (6 ta. edición ed.). Mésxico: Mc Graw Hill.

IIA, I. d.-I.-2. (2004). Marco para la Práctica Profesional Fundación de Investigaciones del IIA. Recuperado el 3 de agosto de 2017, de https://www. csv.go.cr/documents/20126/48202/Consejos+pa$\mathrm{ra}+\mathrm{la}+$ Practica+de+AI.pdf/f515ce27-6cce-f35e-a8ea$81958 c 4 d 2 a 8 a ? t=1558542082573$

IIA, T. I. (2004). iaiperu.org/preguntas-frecuentes/ ique-es-auditoria-interna/. Recuperado el 10 de agosto de 2017, de https://iaiperu.org/preguntas-frecuentes/ ique-es-auditoria-interna/

Instituto Nacional de Estadística e Informática. (2016). Perú en cifras. Obtenido de http://www.inei.gob.pe/

Instituto Nacional de Estadística e Informática, I. (2014). Encuesta Nacional de Satisfacción de Usuarios del Aseguramiento Universal en Salud, 2014. Lima, Lima, Perú. Obtenido de https://www.inei.gob.pe/media/ MenuRecursivo/publicaciones_digitales/Est/Lib1192/

Ley 27785. (22 de julio de 2002). Ley Orgánica del Sistema Nacional de Control y de la Contraloría General de la República. Lima, Lima, Perú. Recuperado el 4 de diciembre de 2019, de http://www.leyes.congreso.gob. pe/Documentos/Leyes/27785.pdf

Lira, J. (2014). Diario Gestión. Obtenido de: https://gestion.pe/economia/pulmon-crecimiento-sector-salud-aseguramiento-privado-68999

Martínez, A., y Campos, W. (2015). Correlación entre Actividades de Interacción Social Registradas con Nuevas Tecnologías y el grado de Aislamiento Social en los Adultos Mayores. Revista Mexicana de Ingeniería Biomédica, Vol. 36(3), 181-191.

Maslow, A. (1991). Motivación y Personalidad. Madrid, España: Ediciones Díaz de Santos S.A. Obtenido de https://www.academia.edu/21608771/ABRAHAM_ MASLOW_-_MOTIVACION_Y_PERSONALIDAD

Piedra-Valoy I, Lipa-Chancolla C. (2018).Calidad de la atención y grado de satisfacción de los pacientes atendidos en el servicio de Emergencia del Hospital Essalud II Vitarte. Rev Soc Peru Med Interna. 31(4):137142 .

Presidencia del Consejo de Ministros, P. (2007). Decreto Supremo 027-2007-PCM. Lima, Lima, Perú. Obtenido de https://es.slideshare.net/yvomiltongaitanlujan/politicas-de-salud-35720456

Resolución de Contraloría No 162-95-CG. Lima, Perú, 2016. Obtenido de https://www.mpfn.gob.pe/escuela/ contenido/actividades/docs/3467_16._nagu_normas_ profesionales.pdf

Resolución de Contraloría 418-2013-CG. Lima, Perú, 2013. Obtenido de Lineamientos de política para la formulación del Plan Nacional Anual 2014: http://www.contraloria.gob.pe/wps/wcm/ connect/29d76738-3ea4-4ceb-b38f-7eff0803c14b/ RC_418_2013_CG.pdf?MOD=AJPERES\&CACHEI$\mathrm{D}=29 \mathrm{~d} 76738-3$ ea4-4ceb-b38f-7eff0803c14b

Vargas, V., Valecillos, J., y Hernández, C. (2013). Calidad en la prestación de servicios de salud: Parámetros de medición. Revista de Ciencias Sociales (RCS), XIX. Número 4, 663-671. doi:http://scielo.sld.cu/scielo.php?script=sci_arttext\&pid=S2077-28742018000100017

Zas, B. (2002). La satisfacción como indicador de excelencia. Obtenido de Psicología-Científica.com: http:// www.psicologiacientifica.com/bv/psicologia 